Collection SFN 9 (2008) 49-64

(c) EDP Sciences, Les Ulis

DOI: 10.1051/sfn:2008005

\title{
Caractérisation des textures par diffraction neutronique
}

\author{
M.-H. Mathon \\ Laboratoire Léon Brillouin, CEA-CNRS, Bât. 563, CEA Saclay, \\ 91191 Gif-sur-Yvette Cedex, France
}

\section{INTRODUCTION}

Les matériaux d'intérêt technologique sont, la plus part du temps, polycristallins c'est à dire constitués d'un assemblage de grains ou cristallites qui sont considérés comme des petits monocristaux. Les propriétés des matériaux dépendent à la fois de la structure de l'agrégat constitué par les grains mais également des propriétés des cristallites. La forme, la taille, leur arrangement au sein de l'agrégat ainsi que leur orientation cristallographique constituent des paramètres déterminant pour les propriétés macroscopiques des matériaux. Lorsque la distribution des orientations de ces cristallites n'est pas aléatoire, on dit que le matériau présente une ou plusieurs orientations préférentielles ou plus généralement une texture cristallographique.

La texture cristallographique est un paramètre microstructural déterminant pour les propriétés (mécaniques, électriques, magnétiques,...) des alliages métalliques via l'anisotropie cristalline (dépendance des propriétés avec la direction cristallographique). La texture apparaît durant la solidification puis évolue au cours des étapes de déformation (laminage, filage, ...) et de recristallisation sous traitement thermique. La compréhension et la maîtrise de l'évolution de ce paramètre au cours de traitements thermomécaniques ou de recuits sont nécessaires pour optimiser le comportement d'un matériau.

Il existe différentes méthodes de caractérisation de la texture : des méthodes basées sur l'imagerie optique comme anciennement les figures de corrosion (obtenues par attaques chimiques des plans de faible indice) ou plus récemment la microscopie électronique à balayage (MEB) ou en transmission couplée avec une analyse EBSD (Electron-Back-Scattered-Diffraction). Ces approches permettent une analyse locale des orientations individuelles $\mathrm{g}_{\mathrm{i}}$. La technique de MEB aujourd'hui automatisée permet une caractérisation de très nombreuses orientations (zones explorées de $10^{4} \mu \mathrm{m}^{2}$ avec un pas de $0,5 \mu \mathrm{m}$ ) et apporte des informations microstructurales complémentaires (désorientations inter et intragranulaires, distribution des joints de grains, localisation des sites potentiels de germination et de croissance...).

Les méthodes de diffraction des Rayons $\mathrm{X}$ ou des neutrons restent les plus utilisées car elles donnent une description globale et statistique de la texture (les effets sont intégrés sur un très grand nombre de grains) et sont plutôt simples à mettre en œuvre. Après un rappel des principales définitions liées à la détermination et à la représentation de la texture cristallographique, la caractérisation par diffraction sera détaillée. L'avantage des neutrons ainsi que les différents aspects techniques de la mesure seront abordés. Enfin, des exemples d'études de textures illustreront d'une part, l'utilisation de la technique de diffraction des neutrons et d'autre part, les corrélations entre la texture et les propriétés des matériaux.

\section{DESCRIPTION DE LA TEXTURE DANS DES MATÉRIAUX POLYCRISTALLINS}

Seules des bases de la représentation et de la détermination des textures cristallographiques seront présentées ici, pour plus de détails, le lecteur pourra se référer aux ouvrages suivants : Bunge (1982), Bunge et Esling (1982), Szpunar (1976).

La caractérisation de la texture consiste à définir l'orientation $(\mathrm{g})$ des cristallites définie par rapport à un référentiel lié à l'échantillon. Ce dernier est déterminé en fonction de la géométrie et de l'historique 


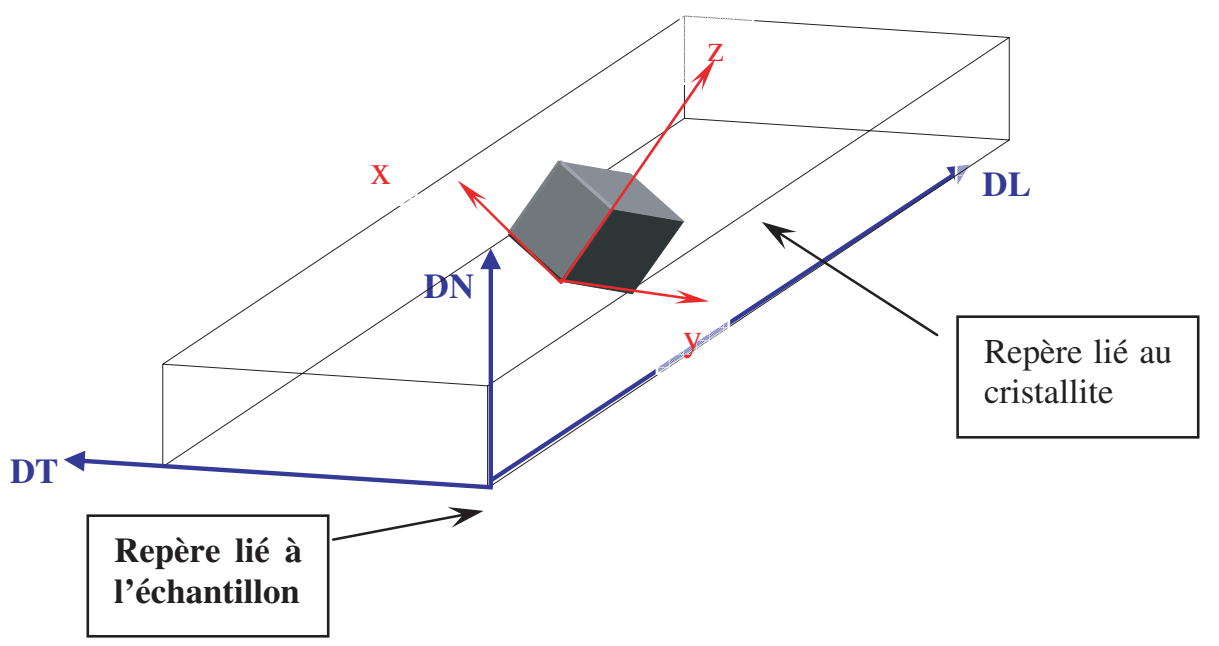

Figure 1. Définition des repères liés à l'échantillon et au cristallite dans le cas d'une maille cubique.

mécanique de l'échantillon. Par convention dans le cas d'une tôle laminée, il est défini par les trois directions suivantes (Figure 1):

DL est la direction de laminage parallèle à $\mathrm{OX}$ (sens de la longueur),

DT est la direction transverse parallèle à OY (sens de la largeur),

DN est la direction normale au plan de laminage (DL, DT) parallèle à OZ.

Le référentiel lié au cristallite (Oxyz) est déterminé en fonction de la symétrie cristallographique. Pour une symétrie cubique, on associera à chaque cristallite le trièdre Oxyz tel que Ox//[100], Oy//[010] et $\mathrm{Oz} / /[001]$. L'orientation du cristallite est définie alors par la rotation g qui permet d'amener en coïncidence, à une translation près, le référentiel Oxyz avec celui lié à l'échantillon.

\subsection{Représentations des orientations préférentielles}

Plusieurs méthodes de paramétrage de cette rotation sont utilisées : matrice de transformation des coordonnées, angles d'Euler, représentation par les indices de Miller etc...Ces deux dernières représentations des orientations sont les plus couramment utilisées en métallurgie.

\subsubsection{Représentation métallurgique}

La représentation de la texture par les indices de Miller est la plus courante. L'orientation cristallographique des cristallites est décrite par:

- la détermination du plan cristallographique $\{\mathrm{hkl}\}$ parallèle au plan de laminage (DL, DT),

- la donnée de la direction cristallographique $\langle\mathrm{uvw}\rangle$ parallèle à la direction de laminage (DL).

Les orientations sont notées $g=\{h \mathrm{hl}\}\langle\mathrm{uvw}\rangle$ (Figure 2). Cette description des textures est qualitativement intéressante car elle indique de façon très claire les orientations principales du matériau mais elle reste insuffisante d'un point de vue statistique et quantitatif. En effet, elle ne fournit aucune information sur l'ensemble de la distribution statistique des composantes : fraction volumique, dispersion etc.

\subsubsection{Utilisation des angles d'Euler}

L'orientation cristalline d'un grain peut être repérée dans le système d'axes lié à l'échantillon par 3 angles d'Euler qui décrivent les trois rotations successives qui amènent en coïncidence les 2 repères. 


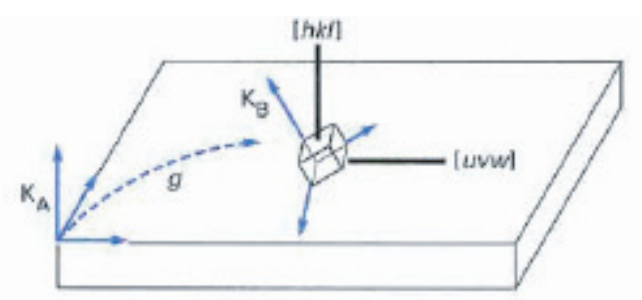

Figure 2. Définition de la représentation métallurgique $\{h k 1\}\langle u v w\rangle$.

Selon les conventions de Bunge, les angles $\varphi 1, \varphi, \varphi 2(0 \leq \varphi 1, \varphi 2 \leq 2 \pi, 0 \leq \varphi \leq \pi)$ correspondent à des rotations respectivement autour de Oz1 (DN), Ox2 et Oz3 (Figure 3).

L'orientation cristalline est alors décrite par ces trois rotations $\mathrm{g}\left(\varphi_{1}, \varphi, \varphi_{2}\right)$. Les 3 angles peuvent être représentés dans un système tridimensionnel de coordonnées cartésiennes ; l'espace ainsi défini est appelé espace d'Euler (ou espace des orientations) dans lequel chaque point représente une orientation cristalline $\mathrm{g}$ à laquelle est associée une densité d'orientation $\mathrm{F}(\mathrm{g})$.
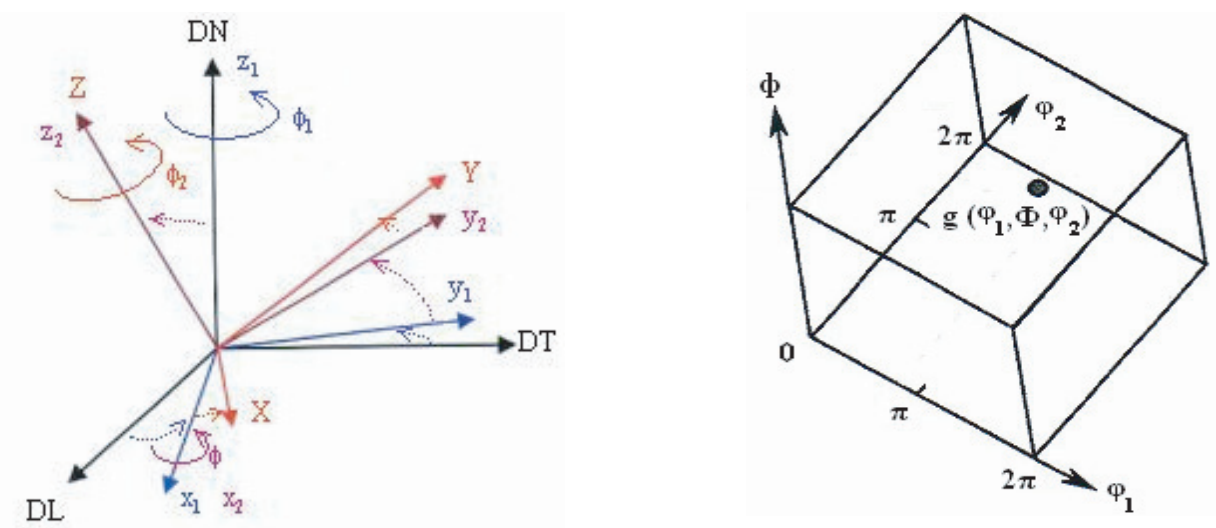

Figure 3. Définition des angles et de l'espace d'Euler.

\subsection{Fonction de distribution des Orientations Cristallines (FDOC)}

La fonction de densité des orientations cristallines est définie par la fraction volumique $\mathrm{dV}(\mathrm{g}) / \mathrm{V}$ des grains ayant une orientation $g$ à dg près :

$$
\frac{\mathrm{dV}(\mathrm{g})}{\mathrm{V}}=f(\mathrm{~g}) \mathrm{dg}
$$

dg est le volume élémentaire dans l'espace d'Euler : $\quad \mathrm{dg}=\frac{1}{8 \pi^{2}} \sin \varphi \mathrm{d} \varphi \mathrm{d} \varphi_{1} \mathrm{~d} \varphi_{2}$.

Cette fonction est positive et normée à 1 . Pour des orientations cristallines aléatoires, à chaque orientation correspond une même fraction volumique; on a donc $\mathrm{f}_{\text {aleatoire }}(\mathrm{g})=1$. La fonction de densité est donc normalisée en multiples de la densité aléatoire et l'unité utilisée est alors le m.r.d. (multiples of the random density). La FDOC est donc une fonction tridimensionnelle représentée dans l'espace d'Euler par des surfaces d'isodensité construites à partir des densités $\mathrm{f}(\mathrm{g})$ associées à chaque point ou orientation $\mathrm{g}\left(\varphi_{1}, \varphi, \varphi_{2}\right)$. Cette fonction fournit une information statistique complète de la distribution 


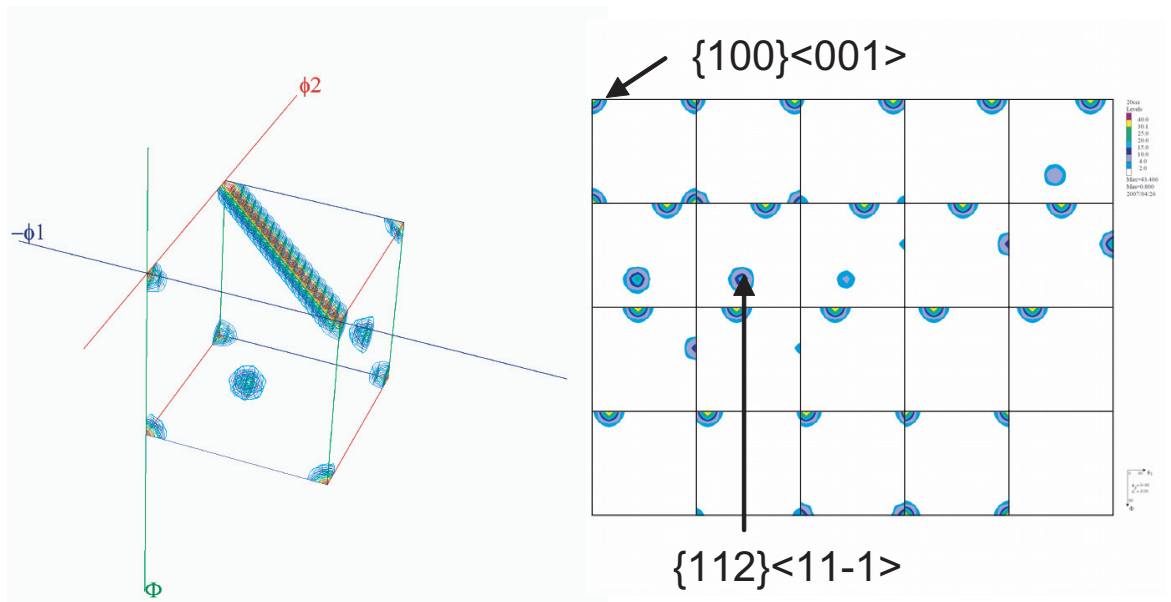

Figure 4. Fonction des distributions des orientations cristallines en représentation $3 \mathrm{D}$ et en coupes à $\varphi_{2}$ constant. Les renforcements observables correspondent aux orientations : $\{100\}\langle 001\rangle$ dite « cube »et $\{112\}\langle 11-1\rangle$ dite « cuivre ».

des orientations cristallines. Son exploitation permet de repérer les orientations préférentielles, les dispersions angulaires autour des orientations idéales, de visualiser les textures de fibres (ensemble continu, etc..) et de quantifier les fractions volumiques des composantes de texture.

Il est usuel de décomposer cette fonction tridimensionnelle en coupes bidimensionnelles (voir en section unidimensionnelle) telles que $\varphi_{1}$ ou $\varphi_{2}$ soit constant, le pas utilisé est généralement de $5^{\circ}$. La Figure 4 donne un exemple de FDOC.

Les orientations cristallographiques préférentielles correspondent aux renforcements de la FDOC et sont désignées en utilisant la description métallurgique, les relations de passage étant:

$\mathrm{h}=\mathrm{n} \sin \phi \sin \varphi_{2} ; \mathrm{k}=\mathrm{n} \sin \phi \cos \varphi_{2} ; 1=\mathrm{n} \cos \phi ; \mathrm{u}=\mathrm{n}^{\prime}\left(\cos \varphi_{1} \cos \varphi_{2}-\sin \varphi_{1} \sin \varphi_{2} \cos \phi\right) ;$

$\mathrm{v}=\mathrm{n}^{\prime}\left(-\cos \varphi_{1} \sin \varphi_{2}-\sin \varphi_{1} \cos \varphi_{2} \cos \phi\right) ; \mathrm{w}=\mathrm{n}^{\prime} \sin \phi \sin \varphi_{1} ;$

$\mathrm{n}$ et n' sont des facteurs pour obtenirdes entiers.

La fonction FDOC ne peut pas être déterminée expérimentalement directement mais elle est calculée à partir des distributions des densités d'orientation bidimensionnelles que l'on peut mesurer, appelées figures de pôles.

\subsection{Figures de pôles}

Une figure de pôles directe (FDP) est la projection stéréographique sur le plan de l'échantillon, de la distribution des densités de pôles d'une famille de plans $\{$ hkl $\}$ dans toutes les directions de l'échantillon. Celles-ci sont données par l'ensemble des couples $(\chi, \varphi)(\chi$ et $\varphi$ sont les angles repérant le plan cristallographique $\{\mathrm{hkl}\}$ par rapport aux axes du référentiel échantillon : $\chi \in\left[0,90^{\circ}\right], \varphi \in\left[0,360^{\circ}\right]$ ) (Figure 5). Le principe de la projection est le suivant : pour un élément cristallin de volume unité donné, on suppose qu'il existe un plan cristallographique $\{\mathrm{hkl}\}$ passant par le centre d'une sphère imaginaire $(\Sigma)$; la normale au plan $\langle\mathrm{hkl}\rangle$ perce la demie sphère de référence en $\mathrm{P} 1$. Le point $\mathrm{P} 2$ intersection de SP1 avec le plan $\pi$ est le pôle du plan réticulaire étudié. Si l'on recommence avec tous les éléments de volume du matériau, on obtient la figure de pôles $\{\mathrm{hkl}\}$ rapportée aux axes liés à l'échantillon. En particulier le plan $\pi$ est, pour une tôle, le plan de laminage. 

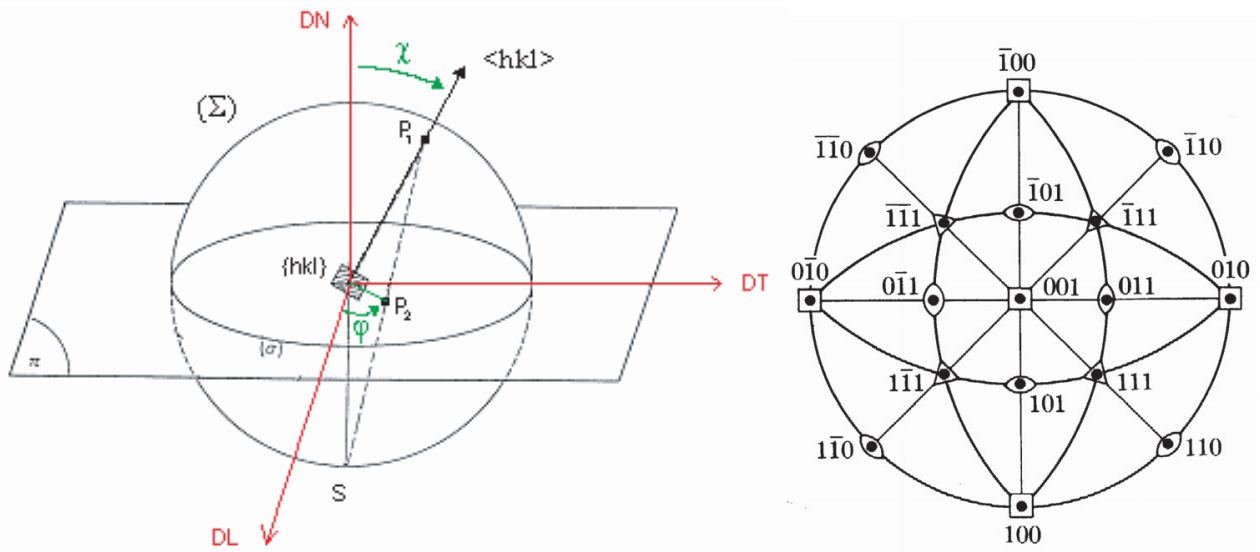

Figure 5. Projection stéréographique : principe et projection $\{100\}$ limitée à $n=1$ d'un cristal cubique.

Sur la Figure 5, est donné un exemple de projection stéréographique de type $\{100\}$. Elle est construite en supposant le cristal orienté tel que le plan [001] est parallèle au plan $\pi$, les pôles reportés correspondent aux projections des plans des familles $\{100\},\{110\}$ et $\{111\}$.

La Figure 6 donne un exemple de figures de pôles expérimentales correspondantes à la FDOC présentée Figure 4 obtenues pour un matériau de symétrie cubique.

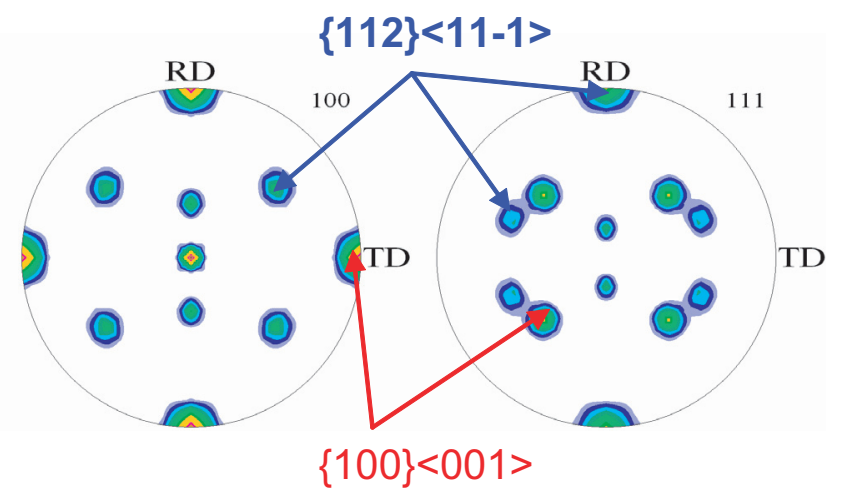

Figure 6. Figures de pôles $\{100\}$ et $\{111\}$ d'un matériau de symétrie cubique correspondantes à la FDOC présentée Figure 4.

Chaque pôle correspond en fait à une multitude d'orientations différentes obtenues par rotation $\gamma$ autour de la direction $\langle\mathrm{hkl}\rangle$. Ainsi, pour une famille de plan $\{\mathrm{hkl}\}$, la densité de pôles en un point $(\chi, \varphi)$ est donnée par l'intégrale :

$$
\mathrm{q}_{\mathrm{hkl}}(\chi, \varphi)=\frac{1}{2 \pi} \int_{0}^{2 \pi} \mathrm{f}(\mathrm{g}) \mathrm{d} \gamma
$$

Cette relation constitue l'équation fondamentale de l'analyse des textures.

\subsection{Détermination de la FDOC}

Deux méthodes mathématiques existent pour résoudre de tels systèmes d'équations intégrales :

1. La Méthode Harmonique

2. La Méthode Vectorielle. 
La méthode harmonique consiste à développer les deux membres de l'équation fondamentale ci-dessus sur des bases d'harmoniques sphériques à un ordre fini $\mathbf{L}$ choisi (dépend de la symétrie du cristal et du nombre de figures de pôles expérimentales) et à trouver des relations entre les deux développements. Elle a été proposée dans les années 1960 par Wiglin (Wiglin, 1960), Bunge [Bunge, 1969] et Roe [Roe, 1965]. La limitation de cette méthode provient des erreurs de troncature qu'elle peut induire et du nombre élevé de figures de pôles expérimentales nécessaires pour les symétries cristallographiques non cubiques. Pour un développement à l'ordre 2, la symétrie cubique requière 3 figures de pôles et la symétrie hexagonale en demande 5.

La méthode vectorielle a été apportée par Williams (Williams, 1968), puis reprise par Ruer et Baro (Ruer et Baro, 1977) et Vadon (Vadon, 1981). Son principe repose sur une discrétisation des figures de pôle d'une part, et des orientations g d'autre part. L'équation fondamentale se transforme en une somme finie d'équations où la densité de pôle $(\chi, \varphi)_{\text {i }}$ connue est approximée par une expression linéaire de densités d'orientation inconnues $\mathrm{f}\left(\mathrm{g}_{i}\right)$. La résolution de ces équations donne une solution discrète de $\mathrm{f}(\mathrm{g})$. Le choix du mode de discrétisation des espaces a conduit au développement de différentes méthodes discrètes ADC (Arbitrarily Defined Cells) (Pawlik et al. 1988) et la méthode WIMV (Williams Imhoff - Matthies - Vinell) (Matthies et Vinel, 1982).

L'avantage de cette méthode est qu'elle nécessite relativement peu de figures de pôles pour le calcul d'une FDOC. Cette dernière méthode donne de meilleurs résultats dans le cas de textures relativement accrus. Pour les textures très marquées, il vaut mieux utiliser la méthode de Helming (Helming, 1994), qui permet de décrire la texture par seulement quelques composantes. Les ajustements sont réalisés sur les figures de pôles en supposant pour chaque orientation idéale une distribution de densité de forme gaussienne définie par son centre, sa largeur à mi-hauteur (dispersion autour de l'orientation idéale) ainsi que son poids.

Lors d'une analyse de texture, deux paramètres sont régulièrement utilisés :

- L'indice de texture (I) qui caractérise l'état global de la texture, toutes orientations préférentielles confondues. Il est égal à 1 pour un état isotrope et augmente avec l'acuité de la texture. Il est égal à :

$$
I=\frac{1}{8 \pi^{2}} \int_{G} f^{2}(g) \cdot d g
$$

où $\mathrm{f}(\mathrm{g})$ est la valeur de la FDOC et $\mathrm{G}$ est l'espace d'Euler.

$\checkmark$ L'erreur relative sur le calcul de la FDOC est estimée à partir de l'équation suivante qui donne l'erreur pour chaque figure de pôles :

$$
\mathrm{RP}\{\text { hkl }\}=\frac{1}{\mathrm{~N}}\left|\sum_{\mathrm{i}=1}^{\mathrm{N}} \frac{\left\{\mathrm{PF}_{\text {exp }}\right\}_{\mathrm{i}}-\left\{\mathrm{PF}_{\text {calc }}\right\}_{\mathrm{i}}}{\left\{\mathrm{PF}_{\text {exp }}\right\}_{\mathrm{i}}}\right| \cdot 100 \%
$$

où $\left\{\mathrm{PF}_{\mathrm{exp}}\right\}_{\mathrm{i}}$ et $\left\{\mathrm{PF}_{\mathrm{calc}}\right\}_{\mathrm{i}}$ sont respectivement les densités de pôles expérimentales et recalculées au même point i, $\mathrm{N}$ est le nombre de points mesurés pour chaque figure de pôles. Puis la moyenne sur l'ensemble des figures donne l'erreur totale sur la FDOC :

$$
R P=\frac{1}{M} \sum_{j=1}^{M} R P\{h k l\}_{j} .
$$

Il est d'usage de ne prendre en compte dans ces différences que les pôles dont la densité est supérieure à un certain niveau. Ce dernier est généralement considéré égal à 1.

\section{PRINCIPE DE LA MESURE DES TEXTURES PAR DIFFRACTION}

La description quantitative des textures nécessite de pouvoir déterminer expérimentalement, pour plusieurs familles de plans $\{\mathrm{hkl}\}$, la densité de pôles en chaque point $(\chi, \varphi)$. La diffraction des rayons 
$\mathrm{X}$ ou des neutrons produit ces informations. La spécificité du rayonnement choisi a des incidences sur la technique de mesures ainsi que sur les applications.

\subsection{Diffraction des rayons $\mathrm{X}$}

La technique des RX peut être employée pour déterminer l'orientation cristallographique grain par grain par la technique de Laue, mais n'est utilisable que dans le cas de matériaux à très gros grains ; l'utilisation la plus courante est la mesure globale par diffraction de poudre.

Le principe de repose sur le fait que l'intensité diffractée par un échantillon polycristallin dépend, entre autres, de la fraction volumique de cristallites en position de Bragg. En présence de texture, cette fraction varie avec l'orientation de l'échantillon par rapport au faisceau incident.

Pour mesurer une figure de pôles correspondante à la famille de plans $\{$ hkl $\}$, l'échantillon, généralement plan, et le diffractomètre sont positionnés pour respecter les conditions de Bragg : le berceau fait un angle $\theta$ avec le faisceau incident et le détecteur est placé à un angle $2 \theta$. Pour explorer la figure de pôles, l'échantillon est installé au centre d'un cercle d'Euler qui assure les rotations $(\chi, \varphi)$. $\chi$ est une rotation polaire comprise entre 0 et $90^{\circ} ; \varphi$ est une rotation azimutale comprise entre 0 et $360^{\circ}$ (voir Figure 7).

L'échantillon est positionné initialement $(\chi=0, \varphi=0)$ avec DT parallèle au faisceau indicent, DN est parallèle à l'axe de rotation $\varphi$. L'intensité diffractée est mesurée pour différentes positions $(\chi, \varphi)$ obtenues par pas successifs $\Delta \chi$ et $\Delta \varphi$ d'environ $5^{\circ}$ (les pas peuvent être resserrés pour des textures très accusées et peu dispersées). Le problème de ce montage est lié au phénomène de défocalisation

(a)
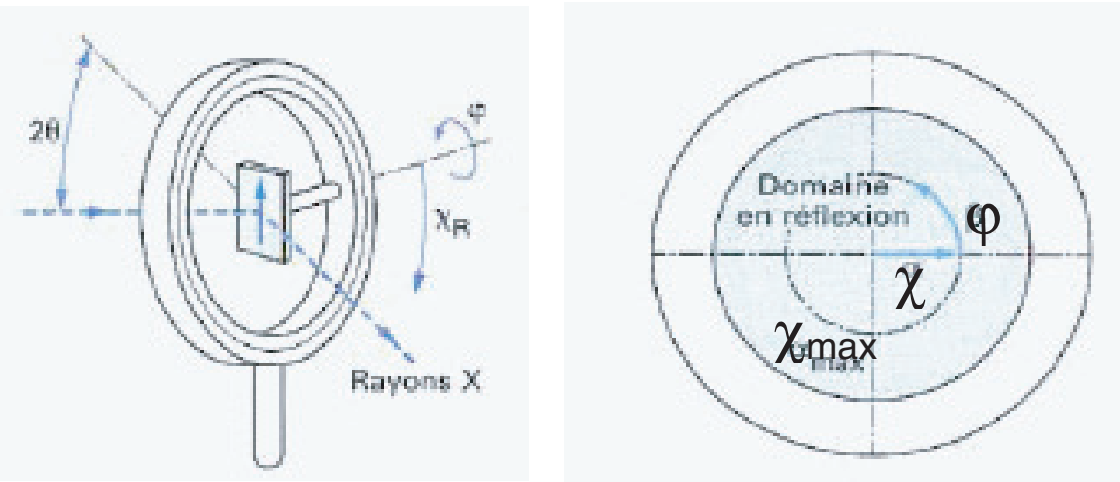

(b)
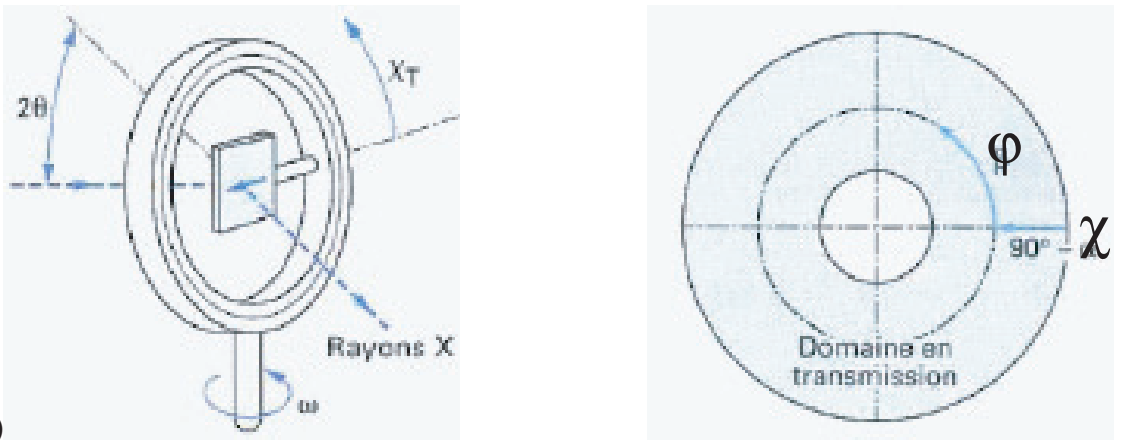

Figure 7. Montage et principe des rotations $(\chi, \varphi)$ pour l'obtention d'une figure de pôles en réflexion. (a) et en transmission (b) par diffraction des rayons X (la flèche représente la direction de laminage du repère lié à l'échantillon) ainsi que représentation du domaine couvert sur la figure de pôles. 
qui correspond à la décroissance de l'intensité mesurée avec l'augmentation de l'inclinaison $\chi$ qui est due à une variation de géométrie du faisceau. Ce phénomène nécessite des corrections importantes des intensités mesurées mais surtout limite les mesures à $\chi=70^{\circ}$. Par conséquent, les figures de pôles obtenues avec ce montage sont toujours incomplètes (voir Figure 7).

Le plus souvent, les figures entières peuvent être reconstruites lors du calcul de la FDOC à partir des mesures en réflexion. Si les périphéries sont importantes pour les interprétations, il faut utiliser alors un montage en transmission pour réaliser ces mesures. Le montage en transmission nécessite d'amincir suffisamment l'échantillon pour qu'une partie du faisceau diffracté soit transmise, compte tenu de l'absorption des rayons X. C'est alors le plan de l'échantillon qui est maintenu parallèle à l'axe $\varphi, \varphi$ étant fixé à 0 pour que la direction normale reste parallèle à l'axe de la rotation $\chi$ (voir Figure 7). La figure de pôles est explorée en faisant varier les angles $\chi$ et $\omega(\theta)$. En transmission, la zone ainsi couverte est limitée à $\chi$ compris entre 60 et $90^{\circ}$, un recouvrement entre les 2 techniques permet de raccorder les intensités relatives à chaque domaine.

La méthode de diffraction des rayons X est la technique la plus courante pour caractériser les textures cristallographiques. Elle est rapide, assez simple à mettre en œuvre dans la majorité des cas si on se limite au domaine de la réflexion. Le temps de comptage pour une orientation $(\chi, \varphi)$ est de quelques secondes ce qui amène le temps de mesure d'une figure de pôles de 1152 points (72 pas en $\varphi \times 16$ valeurs de $\chi$ ) à environ 3 heures. Si le diffractomètre est muni d'un multicompteur, toutes les figures de pôles sont mesurées simultanément.

Néanmoins, les mesures doivent être corrigées de la défocalisation, du volume absorbant puis du bruit de fond (intensité mesurée en dehors d'un pic de Bragg). Ces corrections induisent des imprécisions qui peuvent être importantes. La diffraction de neutrons apporte une meilleure résolution et des avantages supplémentaires.

\subsection{Diffraction des neutrons}

Les avantages de la diffraction des neutrons reposent essentiellement, pour la détermination des textures, sur leur très faible absorption par la plus part des matériaux. Cette propriété permet alors de réaliser les mesures en transmission et d'obtenir des figures de pôles complètes sans autre correction que le bruit de fond. Les erreurs obtenues sur les fonctions de distribution des orientations cristallines sont nettement moins élevées qu'avec des rayons X (le taux d'erreur RP est généralement inférieur à 5\% contre $15 \%$ en moyenne avec les RX). Par ailleurs, la taille des faisceaux de neutrons permet de caractériser des volumes beaucoup plus importants de l'ordre $\mathrm{du}_{\mathrm{cm}}^{3}$ (soit environ $10^{5}$ fois plus grand qu'en RX). Cet atout est indéniable pour :

- l'analyse de matériaux à gros grains $\left(\mathrm{mm}^{3}\right)$,

- l'étude de matériaux qui s'amincissent difficilement comme les matériaux géologiques,

- la caractérisation des phases minoritaires (fractions volumiques de 1\%),

- l'utilisation d'environnements échantillon complexes (four, cryostat, sous chargement etc..). Par exemple, l'évolution de la texture peut être suivie in situ lors d'un traitement thermique.

- l'obtention, dans le cas de matériaux présentant des textures inhomogènes dans le volume, une description globale moyennée sur de larges volumes qui est complémentaire d'une approche locale.

- l'analyse des textures magnétiques.

\subsubsection{Aspect expérimental : un diffractomètre classique}

Sur le plan expérimental, le dispositif est basé sur le même principe de fonctionnement qu'avec des Rayons X. Classiquement, le diffractomètre est équipé d'un berceau d'Euler dans lequel est positionné l'échantillon suivant la Figure 8. Les échantillons ne nécessitent pas de préparation de surface. Leur forme est idéalement sphérique (Tobish et Bunge, 1972) ou cylindrique. Dans le cas de tôles minces, un empilage de tôles collées est utilisé. 


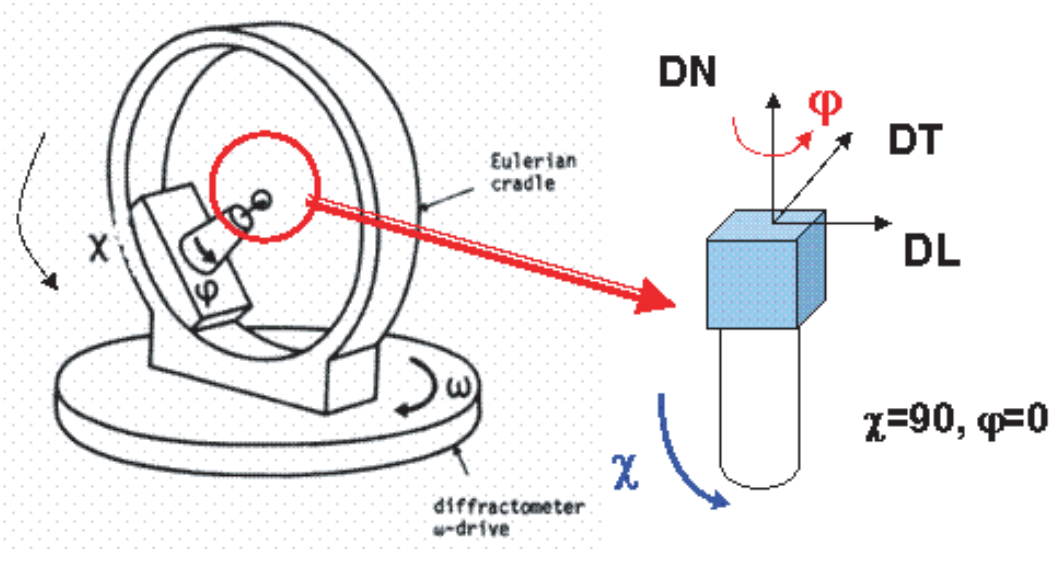

Figure 8. Schéma du berceau d'Euler, des rotations $(\chi, \varphi)$ ainsi que du positionnement de l'échantillon pour des mesures en transmission par diffraction des neutrons.

Le faisceau généralement monochromatique a une section pouvant aller jusqu'à $2 \times 2 \mathrm{~cm}^{2}$. La détection peut se faire avec un monocompteur. C'est la géométrie des 4 cercles 6T1 au LLB et de TEX 2 (Brokmeier, 1997). Dans ce cas, l'acquisition de figures de pôles complètes (1368 points) peut prendre entre 3 et $12 \mathrm{~h}$ selon les collimations utilisées. Ces diffractomètres permettent d'ajuster les paramètres de mesures au système à caractériser (divergence, distance échantillon détecteur, nombre et répartition des points de mesures adaptés à la symétrie de la texture).

La limitation de ces appareils apparaît pour les matériaux de basse symétrie où de très nombreuses figures de pôles doivent être mesurées avec superposition de certains pics.

\subsubsection{Apport des détecteurs PSD}

L'utilisation d'un PSD va permettre comme aux rayons $\mathrm{X}$ de mesurer plusieurs figures de pôles simultanément. En fait, c'est tout le spectre de diffraction qui est obtenu sur un très grand domaine angulaire. Ces données vont permettre de mesurer en un temps bien plus court, un très grand nombre de figures de pôles. Mais surtout, dans le cas où les pics de Bragg sont trop proches pour être séparés expérimentalement, l'analyse du spectre va permettre d'extraire les contributions de chaque famille de plans.

Le principe de la mesure est le même, les différentes orientations de l'échantillon sont obtenues avec le berceau. Ce dernier tourné d'un angle $\theta \mathrm{m}$ ne satisfait la condition de Bragg que pour une seule famille de plans $\{\mathrm{hkl}\}$ dont l'intensité diffusée est recueillie en $2 \theta \mathrm{m}$ sur le multicompteur. Pour les autres angles $2 \theta$, les figures de pôles comportent des zones aveugles aux extrémités. Des mesures complémentaires avec des rotations $(\omega, \chi, \varphi)$ particulières seront nécessaires pour remplir ces manques (Bunge, Wenk, Pannetier, 1982). Ce mode opératoire est utilisé sur le diffractomètre D1B de l'ILL (Grenoble), notamment pour l'étude des roches, des phases intermétalliques ...

Les détecteurs bidimensionnels peuvent également être utilisés pour mesurer simultanément plusieurs points d'une figure de pôles. Le diffractomètre installé à Risø (Juul-Jensen and Kjems, 1983) était conçu sur ce principe. Le détecteur plan était positionné à $2 \theta=90^{\circ}$ (la longueur d'onde étant ajustée pour chaque famille de plans $\{h \mathrm{hl}\}$ ) et orienté verticalement de façon à intercepter une partie du cône de diffraction. Le détecteur mesure alors pour une orientation de l'échantillon, une série de points de la figure de pôles comme représenté sur la Figure 9. La mesure de la figure de pôles complète nécessite beaucoup moins de pas angulaires. Cette technique a permis des acquisitions très rapides de 


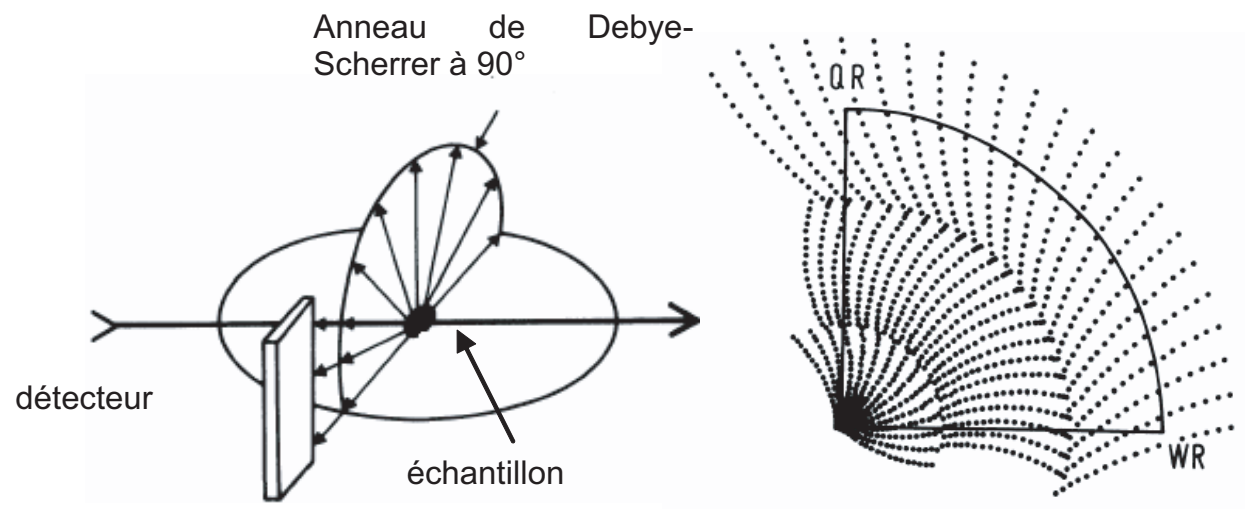

Figure 9. Schéma de Principe d'un diffractomètre équipé d'un multidétecteur vertical positionné à $2 \theta=90^{\circ}$; représentation de l'échantillonnage de la figure de pôle mesurée par petits secteurs angulaires.
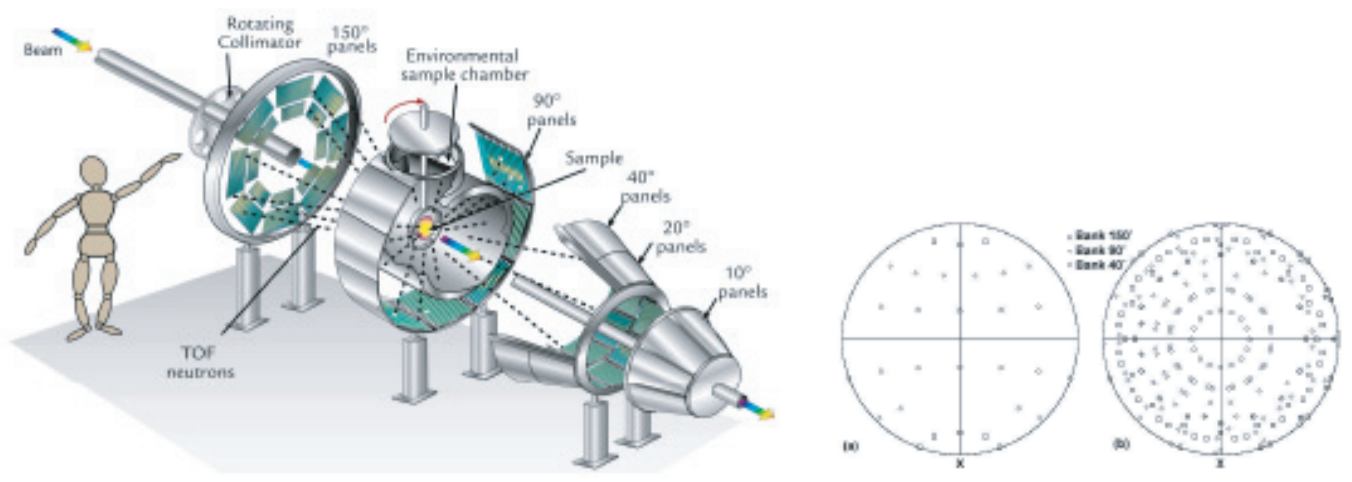

Figure 10. Schéma de principe du diffractomètre HIPPO du LANSCE et point de mesures d'une fdp couverts par les détecteurs situés à $40^{\circ}, 90^{\circ}$ et $150^{\circ}$ pour 1 et 8 orientations de l'échantillon.

figures ou de portions de figures indispensables dans les études in situ de phénomènes de recristallisation ou de transformation de phases.

\subsubsection{Mesures par Temps de Vol}

Ces techniques combinent en fait les PSD avec l'utilisation de faisceaux blancs ou pulsés. On trouve dans cette catégorie des appareils très perfectionnés qui permettent de mesurer plusieurs portions de plusieurs figures en même temps. Le potentiel de ce type de diffractomètre a été montré sur la source pulsée IBR2, à Dubna avec SKAT (Feldmann et al. 1991, Ullemeyer et al. 1998) puis récemment à LANSCE, aux Etats-Unis (H.R. Wenk et al. 2003, S.C. Vogel 2004) avec HIPPO. Cet appareil est constitué de 1360 détecteurs tubes organisés en anneaux et couvrant une surface de détection de $4.8 \mathrm{~m}^{2}$ sur un domaine angulaire compris entre $10^{\circ}$ et $150^{\circ}$ (Figure 10).

La Figure 10 montre également les points de la figure de pôle obtenus sur 3 les anneaux de détecteurs situés à $150^{\circ}, 90^{\circ}$ et $40^{\circ}$ et ce, pour 1 et 8 orientations de l'échantillon. Le temps d'acquisition est alors très fortement réduit et cet appareil est idéal pour les études résolues en temps en température, sous charge extérieure appliquée, etc. 


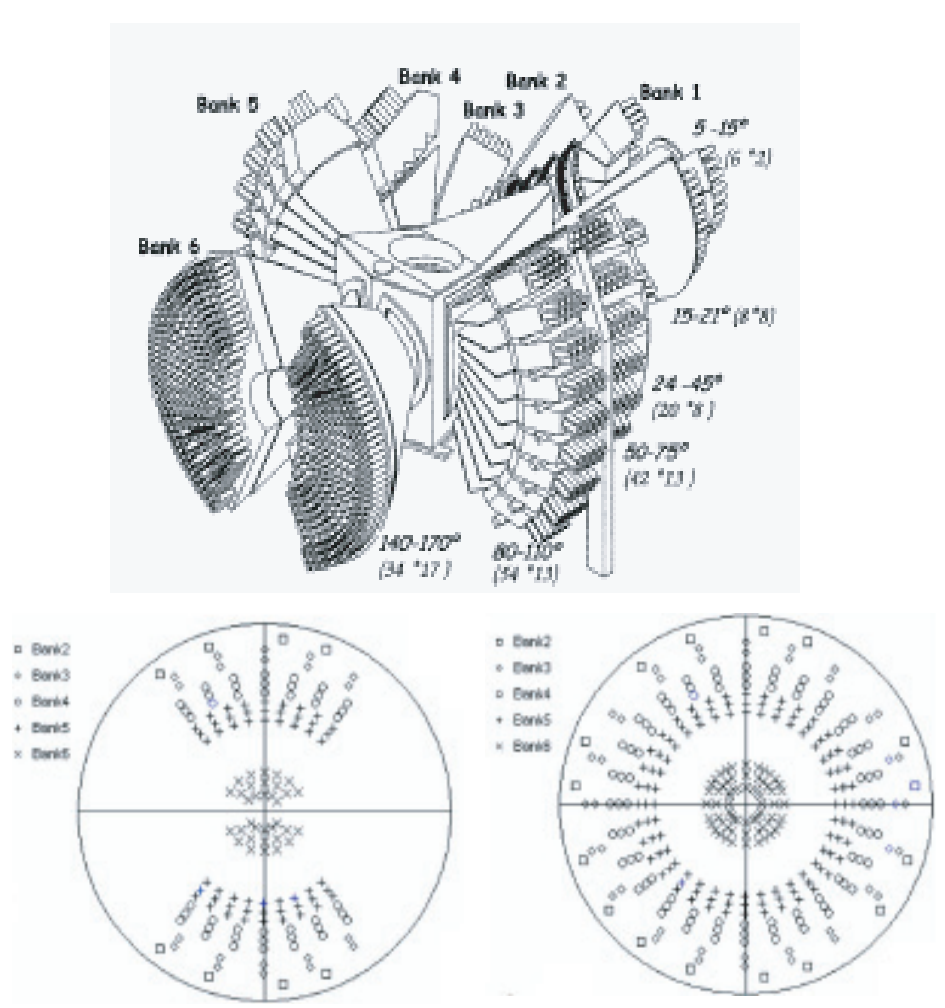

Figure 11. Schéma de principe du diffractomètre GEM à ISIS. Points de mesures d'une figure de pôles obtenus avec l'orientation initiale de l'échantillon puis après une rotation de $90^{\circ}$ autour de la direction du faisceau incident.

Enfin, des analyses de texture réalisées sur GEM à ISIS (Kockelmann et al. 2006) ont montré la puissance de mesures de cette configuration. GEM est un diffractomètre équipé de 6 blocs de détecteurs (7000 détecteurs au total). Pour les mesures de figures de pôles, des groupes de 164 détecteurs sont générés pour couvrir des domaines angulaires de $10^{\circ} \times 10^{\circ}$. La Figure 11 illustre la géométrie de l'appareil et la distribution des points mesurés pour la position initiale de l'échantillon puis après une rotation de $90^{\circ}$ autour de la direction du faisceau incident. 2 ou 3 positions de l'échantillon suffisent pour décrire les figures de pôles correspondant à un temps d'acquisition de l'ordre de quelques minutes !

Le développement de ces appareils a été stimulé par la mise au point de logiciels de traitement de données basés sur la méthode de Rietveld, permettant d'extraire des mesures de temps de vol, les densités d'orientations. En particulier, MAUD (Lutterotti et al. 1997) et GSAS (Von Dreele, 1997) sont utilisés sur GEM et HIPPO.

\section{EXEMPLES D’APPLICATION DE LA DIFFRACTION DES NEUTRONS}

Dans la littérature, un très grand nombre d'exemples d'applications sont disponibles. Les matériaux métalliques font l'objet de très nombreuses études car la texture est un paramètre industriellement très important. Or les évolutions de texture avec le mode de déformation, la composition chimique,...ne sont pas toujours prévisibles. Un exemple de problématique qui se pose dans les alliages CFC est abordé à travers l'étude du FeNi. 


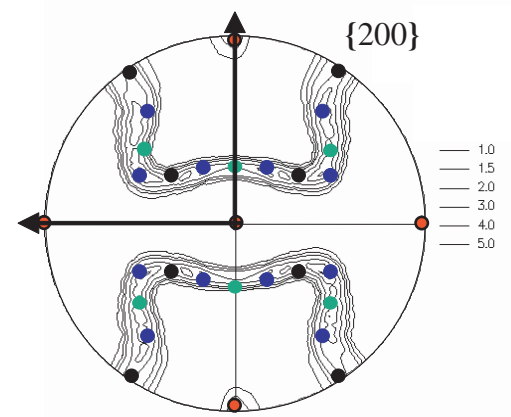

$\operatorname{Max}=5$.

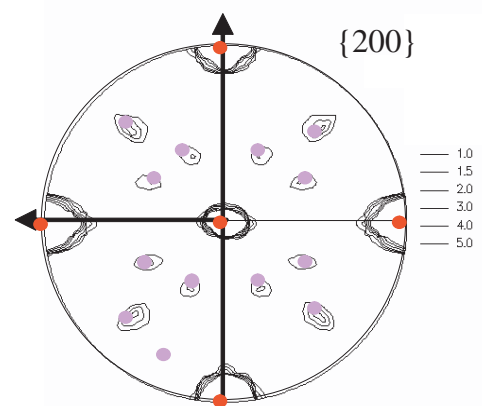

$\operatorname{Max}=47.5$

Figure 12. Figures de pôles $\{200\}$ obtenues sur un alliage FeNi50\% par diffraction des neutrons sur 6T1 au LLB, après un laminage à froid de $95 \%$ et après un traitement de recristallisation total.

\subsection{Déformation et recristallisation d'un alliage CFC : FeNi50\%}

La recristallisation statique des matériaux métalliques lors d'un recuit après déformation conduit à l'apparition d'une nouvelle microstructure ainsi qu'à une modification importante des propriétés mécaniques du matériau. Elle est contrôlée par l'état déformé qui d'un point de vue thermodynamique représente une instabilité due à l'accumulation de dislocations au sein de la microstructure. Bien que présentant un caractère hautement reproductible, les mécanismes régissant le processus de recristallisation après déformation à froid dans les matériaux d'empilement cubique à faces centrées ne sont pas complètement compris (Humphreys, 1995).

L'alliage $\mathrm{Fe} 50 \% \mathrm{Ni}$ de structure CFC est un matériau utilisé principalement pour ses propriétés magnétiques particulières (pôles magnétiques, culasses de relais à haute perméabilité, noyaux de systèmes de sécurité, culasses d'actionneurs spéciaux, transformateur d'impulsion,...). Après un fort laminage à froid et recristallisation primaire, il développe une texture cubique recherchée pour ses propriétés magnétiques particulières.

Comme pour les autres alliages de structure CFC, la texture de recristallisation dépend fortement du taux de déformation. Aux faibles taux de réduction et aux taux de réduction moyens, par laminage à froid, la texture de recristallisation est similaire à la texture de déformation, avec une tendance vers l'isotropie pour les plus faibles taux, alors qu'après une forte déformation, la texture cubique maclée se développe.

Un exemple de cette texture est donnée figure 12 : l'état déformé par laminage à froid jusqu'à un taux de $95 \%$ présente une texture de type cuivre (composée des orientations préférentielles : $\lambda$ $\{123\}\langle 634\rangle$ «Aluminium », $\lambda\{112\}\langle 111\rangle$ «Cuivre », $\lambda\{110\}\langle 112\rangle$ «aiton ») très marquée. Après recristallisation, la composante cube est très marquée accompagnée de sa macle $(\lambda\{001\}\langle 100\rangle$ «Cube », $\lambda\{112\}\langle 221\rangle$ « Macle ») (Branger, et al. 2000).

Afin de comprendre le processus de recristallisation, des mesures in situ à Ris $\emptyset$ sur TXTU ont été réalisées sous recuit à différentes températures entre 500 et $600^{\circ} \mathrm{C}$. L'évolution des pôles caractéristiques des orientations préférentielles a été suivie avec une résolution temporelle de l'ordre de quelques secondes pour les premiers stades. Ces expériences ont permis de décrire les cinétiques de disparition des composantes de déformation et d'apparition de la composante cube. Ces deux phénomènes sont parfaitement simultanés. La Figure 13 présente les intensités mesurées représentées sous forme de facteurs d'avancement de la réaction. A $580^{\circ} \mathrm{C}$, les évolutions des composantes de déformation (Cuivre, Laiton et Aluminium) et de recristallisation (Cube et Macle) sont parfaitement simultanées. Ce résultat suggère que le développement de la composante cube ne serait pas régit par un mécanisme de croissance orientée, explication très souvent avancée dans la littérature. 

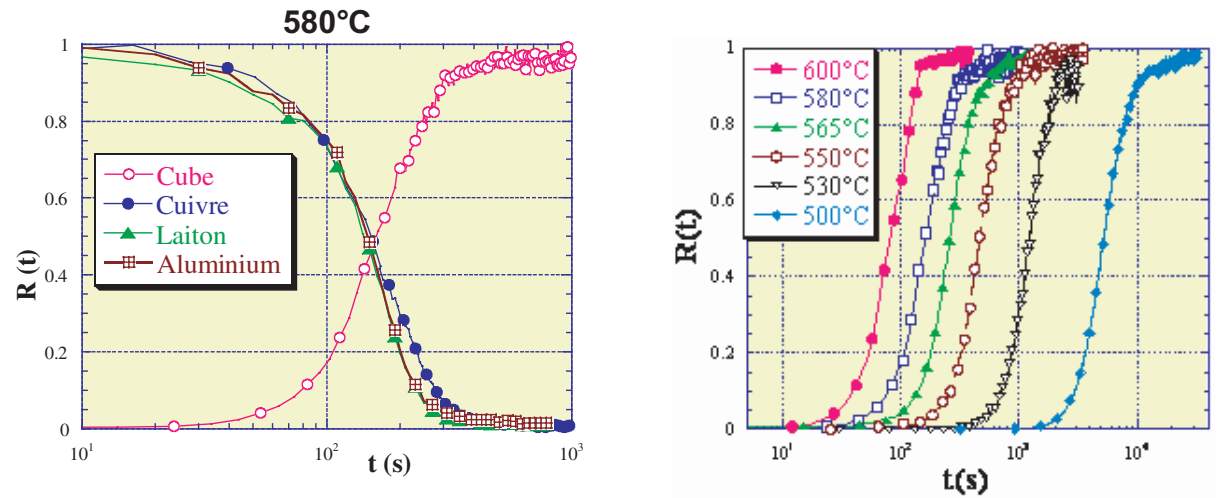

Figure 13. Cinétique de recristallisation mesurées par diffraction des neutrons an suivant l'évolution des intensités diffractées relatives aux pôles caractéristiques de chaque orientation préférentielle liée à la déformation et à la recristallisation (a) sous recuit à $580^{\circ} \mathrm{C}$ et évolution de la composante « cube » à différentes températures (b).
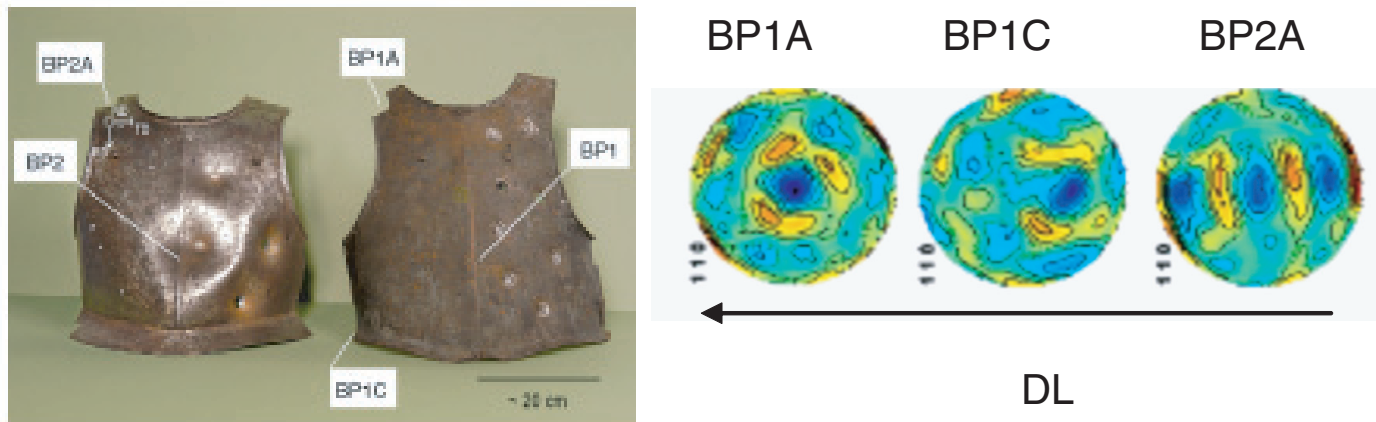

DL

Figure 14. Photographies des cuirasses dénommées BP1 et BP2 avec repérage des zones étudiées et du repère choisi (DL prise dans le sens de la hauteur, DN est normale à la surface).

Par ailleurs, les mesures de cinétique de formation de la composante cube pour des températures de recuit comprises entre $500^{\circ} \mathrm{C}$ et $600^{\circ} \mathrm{C}$ ont permis de déterminer une valeur de l'énergie d'activation de $2.5 \pm 0.2 \mathrm{eV}$.

\subsection{Texture d'une cuirasse d'un arquebusier (Leever et al. 2006)}

Deux cuirasses d'arquebusier (Figure 14) supposées datées du $16^{\text {ème }}$ ou $17^{\text {ème }}$ siècle mais de provenances très différentes, ont vu leurs compositions chimiques et leur textures analysées par diffraction des neutrons en temps de vol sur ROTAX et GEM à ISIS.

La diffraction de neutrons a montré que la phase dominante est la ferrite avec présence de phases minoritaires : la cémentite $\left(\mathrm{Fe}_{3} \mathrm{C}\right)$, la magnétite $\left(\mathrm{Fe}_{3} \mathrm{O}_{4}\right)$ et $\mathrm{FeO}$. La distribution de carbone dans la cuirasse $\mathrm{BP} 1$ est très hétérogène et laisse penser à une carburation accidentelle sur une face. $\mathrm{BP} 2$ ne présente ni cémentite ni magnétite.

L'analyse de la texture de BP1 montre une répartition aléatoire des renforcements en fonction du point de mesure, alors que dans $\mathrm{PB} 2$, la texture est plus régulière et s'apparente à une texture de laminage propre au fer cubique centré.

Les armuriers du $16^{\text {ème }}$ siècle, fabriquaient ces objets par martelage du fer sous forme de feuillards. Ils forgeaient plusieurs feuilles en même temps pour augmenter l'épaisseur. Il n'y avait aucun traitement volontaire de durcissement de ces matériaux. Le laminage n'existant pas à cette époque, ces résultats 
démontrent donc que la cuirasse BP2 est plus récente. Ceci est confirmé par la présence de Mn en teneur trop importante pour cette époque. Cette cuirasse serait une réplique pouvant dater de 1850-1900.

\section{3 Étude in situ de l'évolution de la texture de la glace soumise à un chargement uniaxial (Mc Daniel et al. 2006)}

Ce travail a nécessité le développement d'un nouveau dispositif permettant de déformer la glace à basse température et adapté, entre autres, au diffractomètre HIPPO. L'objectif de l'étude étant de corréler les évolutions de texture à la contrainte appliquée.

Un échantillon polycristallin de $\mathrm{D}_{2} \mathrm{O}$ de forme cylindrique $(2.23 \mathrm{~cm}$ de haut et $2.54 \mathrm{~cm}$ de section) a été utilisé pour éviter les inconvénients de l'hydrogène et les corrections d'absorption. Le dispositif permet d'appliquer une pression sur le haut de l'échantillon avec un piston qui est compressé avec un chargement de tôles (Figure 15a). La charge maximale est de 667N. Le dispositif après chargement est inséré dans la chambre échantillon du diffractomètre (Figure 15b).

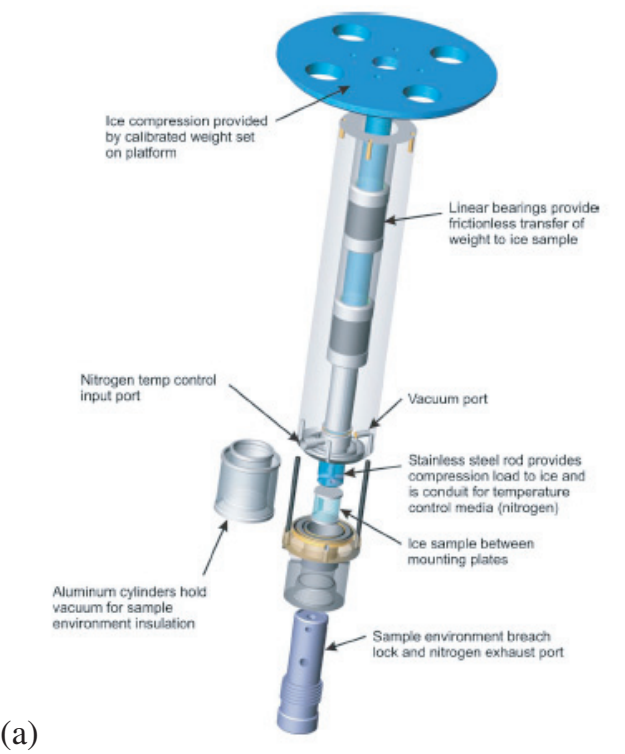

(b)

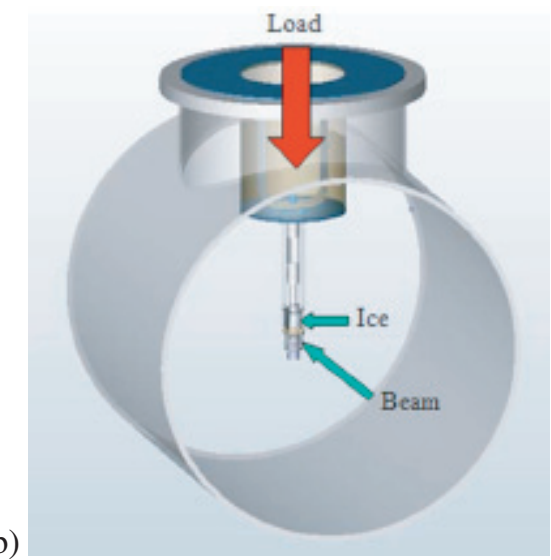

Figure 15. Schéma du dispositif permettant de déformer, en compression, un échantillon maintenu à basse température. (a) et de son adaptation dans la chambre échantillon du diffractomètre HIPPO.

Un chargement de $20 \mathrm{~kg}$ a induit une réduction de la longueur de l'échantillon de $0.2667 \mathrm{~cm}$, soit une déformation totale de 0.210 . Les figures de pôles $\{10-10\},\{0002\},\{10-11\}$ et $\{10-12\}$, propres à la symétrie hexagonale de la glace, ont été mesurées, après stabilisation de la température (en 148 minutes), avec des intervalles de 5 minutes sur une durée totale de 7.5 heures sous charge. Durant cette période, la température a varié entre 230 et $240 \mathrm{~K}$. Une série de figures de pôles est présentée Figure 16. La direction de sollicitation est normale au plan des figures de pôles. Une texture de fibre de type $\{0002\}$ est clairement visible, ce qui signifie que les plans de base $\{0002\}$ sont perpendiculaires à l'axe de compression. Cette orientation préférentielle est usuellement rencontrée dans les matériaux hexagonaux métalliques. En revanche, aucune évolution n'a été observée entre 148 minutes et 7.5 heures de chargement. Ce résultat est un peu surprenant et nécessite une caractérisation de l'état initial hors charge avant toute interprétation.

Ces premières expériences ont eu le mérite de montrer la faisabilité de suivre la texture à différentes températures couplées à une déformation en compression avec une résolution temporelle de quelques 


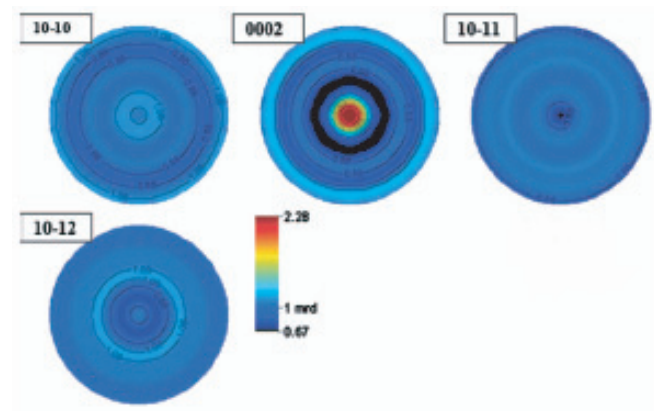

Figure 16. Figures de pôles mesurées sur un échantillon de glace soumis à une compression uniaxiale parallèle à DN après 148 minutes et à une température de $230 \mathrm{~K}$.

minutes. Cette démarche devrait trouver de nombreuses applications en science des matériaux, en particulier dans le domaine de la géologie.

\section{CONCLUSION}

Ce document a pour objectif de donner quelques connaissances de base sur la texture cristallographique : sa définition, les conventions et nomenclatures ainsi que les techniques de caractérisation expérimentales. La diffraction des neutrons offre des possibilités inégalées à ce jour pour étudier des matériaux ayant une microstructure particulière (à très gros grains, multiphasés, hétérogènes, phases minoritaires, textures magnétiques, ...) ou ne se prêtant que difficilement aux mesures par rayons X. C'est le cas des matériaux géologiques ou des matériaux d'origine minérale pour lesquels la diffraction des neutrons par technique du temps de vol apporte une caractérisation en volume de la texture de chacune des phases présentes avec des temps de mesures très courts. Cet aspect est fondamental pour les matériaux de basse symétrie pour lesquels de nombreuses figures de pôles doivent être mesurées. Le lecteur pourra trouver des exemples de travaux dans lesquels l'apport des neutrons est très bien illustré. Günther (2002) s'est ainsi intéressé aux corrélations entre la texture cristallographique et les propriétés magnétiques dans un minerai de fer. Pyzalla et al. (2006) ont publié une étude originale sur des os fossilisés de dinosaures.

Par ailleurs, cette technique permet une description statistique de la texture plus précise que les autres techniques. La précision sur la FDOC est excellente permettant d'une part, de suivre de très faibles évolutions et d'autre part, d'utiliser ces données expérimentales comme paramètres d'entrée dans des modélisations.

Dans le domaine de la science des matériaux, un grand nombre de travaux ont pour objectif de décrire et d'optimiser le comportement macroscopique des métaux et alliages, à partir de la caractérisation et de l'analyse de leur microstructure et de leurs mécanismes fins de déformation. Il est ainsi important de caractériser les hétérogénéités de comportement à l'échelle du grain (qui relève du domaine du synchrotron garantissant une résolution spatiale intragranulaire), des phases ou bien des orientations cristallographiques. A ces échelles, la diffraction des neutrons apporte des informations statistiques moyennées sur un grand nombre de grains. Les scientifiques ont alors besoin de mesurer la texture, les déformations, voir les transformations de phase in situ sous charge et/ou en température pour suivre de façon continue, le comportement sous sollicitations extérieures, avec une résolution temporelle compatible avec les échelles de temps des phénomènes.

\section{Références}

[1] Branger V., Mathon M. H., Baudin T. and Penelle R., Scripta Mater. 43, 325 (2000).

[2] Brokmeier H. G., Physica B 234, 1144 (1997). 
[3] Bunge H. J., Mathematische Methoden der Texturanalyse (Akademie-Verlag, Berlin, 1969).

[4] Bunge H. J., Texture Analysis in Materials Science (Butterworths, London, 1982).

[5] Bunge H. J., Esling C., “Quantitative Texture Analysis”, Bunge and Esling Ed., DGM (1982).

[6] Bunge H. J., Wenk R. and Pannetier J., Textures and Microstructures, 5, 153 (1982).

[7] Feldmann K., Betzl M., Kleinsteuber W., Wakher K., Textures and Microstructures, 14-18, 59 (1991).

[8] Günther A., Brokmeier H. G., Petrovsky E., Siemens H., Helming K. and Quade H., Appl. Phys. A 74, 1080 (2002).

[9] Helming K., Materials Science Forum 157-162, 363 (1994).

[10] Humphreys F. J. and Hatherly M., Recrystallization and Related Annealing Phenomena (Pergamon Press, 1995).

[11] Juul-Jensen D. and Kjems J. K., Textures and Microstructures 5, 239 (1983).

[12] Kockelmann W., Chapon L. C. and Radaelli P. G., Physica B 385-386, 639 (2006).

[13] Leever S., Visser D., Kockelmann W. and Dik J., Physica B 385-386, 542 (2006).

[14] Lutterotti L., Matthies S., Wenk H. R. and Vinel G., J. Appl. Cryst., 21, 285 (1988).

[15] Matthies S. and Vinel G. W., Physica Status Solidi. 112 (1982).

[16] Pawlik K., Pospiech J. and Lücke K., Eight International Conference on Textures of Materials (ICOTOM 8), Mexique, TMS Warrendale, 105 (1988).

[17] Pawlik K. and Pospiech J., Lücke K., Textures and Microstructures, 14-18, 25 (1991).

[18] Roe R. J., J. Appl. Phys., 36, 187 (1965).

[19] Pyzalla A. R., Sander P. M., Hansen A., Ferreyro R., Yi S. B., Stempniewicz M. and Brokmeier H. G., Mat. Sci. Eng. A 437, 2 (2006).

[20] Ruer D. and Baro R., Adv. X-ray Anal. 20, 187 (1977).

[21] Szpunar J., Atomic Energy Review, 14, 199 (1976).

[22] Tobisch J. and Bunge H. J., Texture 1, 125 (1972).

[23] Ullmeyer K., Spalthoff P., Heinitz J., Isakov N.N., Nikitin A.N. and Weber K., Nucl. Instrum. Meth. Phys. Res. A 412, 80 (1998).

[24] A. Vadon, Thèse de doctorat, Université de Metz (1981).

[25] Vogel S. C., Hartig C., Lutterotti L., Von Dreele R. B., Wenk H. R. and Williams D. J., Advances in X-ray Analysis 47, 431 (2004).

[26] Von Dreele R. B., J. Appl. Cryst. 30, 517 (1997).

[27] Wenk H. R., Lutterotti L. and Vogel S., Nucl. Instrum. Meth. A 515, 575 (2003).

[28] Wiglin A. S., Soviet. Phys. Solid. State 1, 229 (1960).

[29] Williams R. O., J. Appl. Phys. 39, 4329 (1968). 